\section{Distributions of Invasive Weed Parthenium (Parthenium hysterophorus L.) in the University Campus Peshawar, Pakistan}

\section{Abstract}

A survey was performed to measure the distribution of invasive parthenium ( $P$. hysterophorus) weed in the university campus, Peshawar, the capital city of Khyber Pakhtunkhwa province in 2016. The University Campus included: The University of Peshawar, Islamia College University, The University of Agriculture Peshawar, Pakistan Forest Institute and the New Developmental Research Farm, The University of Agriculture Peshawar were the main studied areas. In the selected areas $P$. hysterophorus distribution was measured in the academic, residential and research farm areas. Moreover, the survey also investigated the impact of parthenium weed upon native weeds flora inside the University campus. A $1 \mathrm{~m}^{2}$ quadrat was used to collect the data. During the survey, a total of 32 weeds species were recorded belonging to 18 different families and 32 genera, the life cycle of 23 weeds were annual while 9 weeds have perennial life cycle. The data were recorded on absolute density (\%), relative density (\%), absolute frequency (\%), relative frequency (\%) and importance values (\%) of parthenium weed and others flora. The data showed that parthenium weed was most dominant species having $76.8 \mathrm{~m}^{-2}$ plants density in the NDF and $\left(32.0 \mathrm{~m}^{-2}\right)$ plants in Islamia University. However, the highest frequency $(100 \%)$ of $P$. hysterophorus was observed for The University of Agriculture, while minimum (80\%) occurred in Agronomy field. Moreover the relative frequency of $P$. hysterophorus was maximum (25\%) seemed for Islamia College; whereas, its minimum (12.9\%) at Agronomy field. Lastly, the Importance Value of Parthenium indicates the highest $(45.98 \%)$ at Dairy farm followed by $(40.33 \%)$ at Horticulture field's area. In conclusion, the Parthenium weed become more problematic and replaces all the native flora in the non-crop area specially Dicanthium annulatum a native grass specie on the on the ridges of the field being replaced by parthenium. Due to no proper management and high growth rate, nowadays it becomes as super weed in the KPK and especially in the Pakistan. So, the management of Parthenium weed requires the call attention from the Govt. policy maker and proper quarantine inspection to conserve the native flora and field crops to get maximum crop yield.

Keywords: Parthenium hysterophorus; Weed frequency; University campus; Native flora

\author{
Shah Zareen ${ }^{1,2 *}$, \\ ljaz Ahmad ${ }^{2}$, Akhtar Ali ${ }^{1}$, \\ Haris Ali Khan', \\ Irfanullah Khan', \\ Muhammad Fawad ${ }^{2}$ and \\ Said Rahman²
}

1 Department of Biomedical Sciences and Engineering, Konkuk University, Seoul, South Korea

2 Department of Weed Science, The University of Agriculture Peshawar, Pakistan

\section{*Corresponding author: Shah Zareen \\ ” shahzareen75@gmail.com}

Department of Biomedical Sciences and Engineering, Konkuk University, Seoul, South Korea.

Tel: +8201032654275

Citation: Zareen S, Ahmad I, Ali A, Khan HA, Khan I, et al. (2018) Distributions of Invasive Weed Parthenium (Parthenium hysterophorus L.) in the University Campus Peshawar, Pakistan. Eur Exp Biol. Vol.8 No.3:18

\author{
Abbreviations: D.F.A: Dairy Farms Area; A.F.A \\ Agronomy Farm Area; P.B.G.F: Plant Breeding Genetic Farm; \\ H.F.A: Horticulture Farm Area; N.A.B: New Academic Block; \\ F.I.C: Forest Institute Colony; F.I.J: Forest Institute Jangle Area; \\ D.G: Dhobi Guard; I.C.S: Islamia Collegiate School; I.C.U: Islamia \\ College University
}

\section{Introduction}

Parthenium hysterophorus $L$. weed is an annual plant belongs to family Asteraceae. The plant can achieved height up to 2 meters when germinated in healthy soil and also have the ability to produce flowers after 4-6 weeks of germination [1]. A healthy and mature plant can produce up to 15000 seed plant-1 which 
could be transferred through moving water, animal's body, clothing vehicles, winds, machinery or birds [2]. In favorable environmental conditions, mostly the plant seeds germinate in two years [3], even though an important part of buried seeds have the ability to endure as dormant for numerous years [4]. For healthy germination of requiring alkaline to neutral clay soils [5], while could be grown on less effective on a various kind of soil natures. The water availability for this weed growth and germination comparatively more are inadequate by deprived rainfall [6].

Parthenium is a noxious and aggressive weed to South America and central tropical, and now days spreading to the subtropical and tropical content of the world [7]. In 1956, by chance the parthenium weed was introduced in India [8] and nowadays found everywhere of Indian sub-continent countries, like Pakistan. In Asia, the spreading of Parthenium weed very fast and reached to Vietnam, Taiwan and southern China [9], also reported in African countries like; South Africa, Kenya, Mozambique and Ethiopia and invasive for many Pacific Islands $[10,11]$.

The invasive Parthenium creates problems for human health as well losses of agriculture yields. The Parthenium hysterophorus $L$. plant and its relative species have sesquiterpene lactones [12] which encourage the allergic dermatitis problems and other signs. Create problems as damage to human health, crop production, biodiversity and animal husbandry throughout the globe [13]. The invasive structure of Parthenium replacing the endangered local medicinal plant flora which utilized as medicine source [14]. Especially in India, Parthenium reduces the several crop yields up to $40 \%$ [15] while, up to $90 \%$ losses in forage production [9]. Another hand in Australia, found as a major threat to perennial grasslands in the central state of Queensland [1,4] whereas losses in beef production up to AU\$22.0 million per year [16]. Parthenium, now introduces himself as cropland weed in Pakistan [13]. For domestic animals it is inedible, but sometimes sheep and cattle will eat in feed limitation or rare.

Globally, the high costs of herbicides prohibit their use for Parthenium weed control. Management will be a success when growing fine pasture grass which has the capability to compete with Parthenium. In the more severe situation of the crop, Parthenium weed can be controlled by application of 2, 4- D or Atrazine [17]. Bio-agents is well substituted to the chemical for perennial grasslands, which is the natural management of Parthenium could be in progress especially in Australia etc.

This survey looks at the current occurrence of Parthenium weeds inside the primitivity of Peshawar University Campus and in the New Developmental Research Farm, The University of Agriculture Peshawar, Pakistan.

In spreading, soil as seed banks is reported for seed dormancy, seed longevity and the capability of weed to grow in the favorable environmental conditions. From the previous investigation, the dormancy of seeds in soil was resolute to be high (44639 seeds $\mathrm{m}^{-2}$ ) Queensland [18] and soil seed bank persistency reached to $65-87 \%$. The seedling of Parthenium invasive weed rapid growing observed from soil samples as compared to other plants species. Keeping in view the importance of this invasive weed in term of suppressing native flora a comprehensive survey was conducted in the University campus with the following objectives.

- To identify the weed flora of University campus, Peshawar

- To find out the most infested university among the three studied universities regarding weeds population

- To compute the infestation of invasive Parthenium weed around the University Campus, Peshawar.

\section{Materials and Method}

A survey was designed to investigate the Parthenium weed distribution in The University campus Peshawar Khyber Pakhtunkhwa, during November 2016. The survey sites, University Campus and The New Developmental Farm, the University of Agriculture Peshawar were selected. Two residential sectors, the University Campus and The New Developmental Farm (NDF) were randomly selected. In the surveyed areas, Parthenium weed was mapped in occurrence and distribution of wastelands as well crop field's flora. A study was conducted in order to study the influence of Parthenium on the occurrence of native weeds inside the Campus and New Developmental Farm, the University of Peshawar. The data collection was done from undisturbed zones along the field and vacant plots. Data were collected from various selected points inside the Campus (University of Peshawar, Islamia College University and The University of Agriculture) and inside the New Developmental Farm. At each point, five quadrats $\left(1 \mathrm{~m}^{2}\right)$ were randomly placed and each time Parthenium distribution, as well as other plant species coming inside the quadrants, were counted and recorded. Frequency and density of Parthenium and other plant species were estimated by using the following formula [19].

Absolute Density, $\mathrm{AD}(\%)=\frac{\text { Total number of individualof a particular species in all quadrants }}{\text { Total number of quadrats placed }}$

Relative Density, $\mathrm{RD}(\%)=\frac{\text { Absolute density for a specific species }}{\text { Total absolute density for all species }} \times 100$

Absolute frequency, $\operatorname{AF}(\%)=\frac{\text { Number of quadrats in which species occurs }}{\text { Total number of quadrats }} \times 100$

Relative frequency, $\operatorname{RF}(\%)=\frac{\text { Absolute frequency value for a specific species }}{\text { Total absolute frequency values for all species }} \times 100$

Importance value, $\operatorname{IV}(\%)=\frac{\mathrm{RF}+\mathrm{RD}}{2}$

In selected locations, two areas along the field were randomly selected and then surveyed. The placement of five $\left(1 \times 1 \mathrm{~m}^{2}\right)$ quadrats was randomly done in a horizontal pattern, in each location. In placed quadrats, the spaces among each quadrate were depending upon the availability of the area along the field and any obstructions that may have been present. The identification of collected weed was done in the Department of Weed Science, The University Of Agriculture Peshawar, Pakistan (Figure 1). 


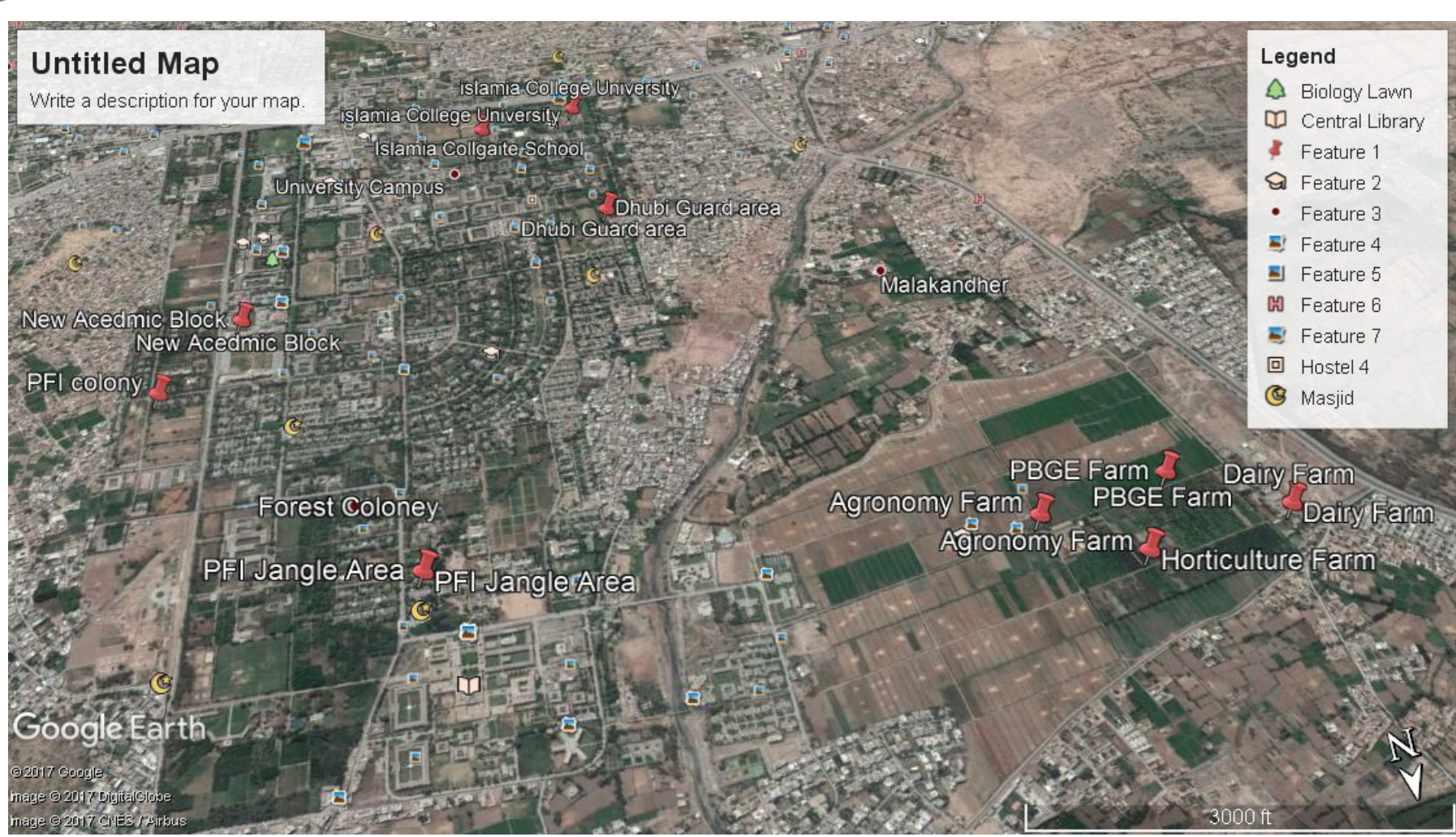

Figure 1 Map with GPS Coordinates of the (studied area), where the distribution of parthenium and other weeds flora in the university campus, Peshawar.

\section{Results and Discussion}

Interestingly in the total of 32 Weeds species were identified and collected, belonging to 18 families and 32 genera. 23 weeds have annual life cycle and 9 weeds have perennial. Distribution of annual weed nature shows that few of the annual weeds were found the road sides while majority in the fields as the field have average rotation of two crops mostly which flourish the growth of annual weeds while the perennial weeds mostly grown on the ridges, the only dominant species in all the field area non field area mostly ridges was parthenium which mostly displayed the native species specially Dicanthium annulatum a native weeds specie become extinct now a days on the ridges and road sides. Moreover the study also shows that parthenium is susceptible to field disturbance and other field relative operations i.e tillage, ploughing. Most of the crop with great density have no parthenium weed recorded while crop growing on the ridges with free spaces invaded by parthenium i.e maize fields. The overall distribution and frequency of all the weeds recorded were as shown in the table listed below (Table 1).

\section{Absolute weed density}

The data in illustrates that the variability in weed dynamics throughout the 10 studied sites. In absolute weed density resultant data, the utmost distribution of Parthenium weed (76.8 $\mathrm{m}^{2}$ ) was confirmed at Dairy farm, the University of Agriculture. Whilst, the distribution of Parthenium at Agronomy field, New Academic Block, Horticulture fields, and Plant Breeding and Genetic engineering fields were infested with the amount of
(59.2, 58.4, 50.4 and $44.8 \mathrm{~m}^{2}$ ) respectively. However, except of Parthenium weed, the infestation of Cynodon dactylon L., at Islamia Collegiate School with 100 plants $\mathrm{m}^{2}$ were noted. In studied sites, the calculated mean of all weed species bring to light, the Cynodon dactylon L. $50.56 \mathrm{~m}^{2}$ was most predominant species. Apart from that, the Parthenium $\left(48.08 \mathrm{~m}^{2}\right)$ and Oxylis weed species infesting to the extent of $10.48 \mathrm{~m}^{2}$ and all other areas which possessed the equal number. Moreover, the lowest absolute weed density in studied sites occurredat the Islamia Collegiate School, Islamia College University, Daubi Guard and New Academic Block as the amount of $(32,36.8,38.4$ and 41.6 $\mathrm{m}^{2}$ ). The Parthenium weed acceleration was observe in noncropping area as well up to some extend in agriculture growing sites which inhibits the crop yields as well as replace other native flora. These findings are agreement of $[20,21]$ who report that the distribution of invasive Parthenium, damage the agriculture crops and its production as well as agitate the ecosystem and cause human health (Table 2 ).

\section{Relative weeds density (\%)}

As regards to relative weed density data (\%) of all studied sites at the University Campus Peshawar, The aggrandize percent of relative weeds density (48.48\%) for Dairy farm area, (35.20\%) Horticulture field area, New Academic Blocks (33.33\%), Agronomy field area (30.19\%) and Forest Institute jungle area (25.24\%) of flora comprised of Cynodon dactylon L. weed species respectively. However, relative density even surpassed the Cynodon dactylon L. at Islamia College University (36.41\%) and Forest Institute colony 
Table 1 Taxanomy and life cycle of collected and identified weeds in the university campus.

\begin{tabular}{|c|c|c|c|c|}
\hline S.No & Scientific name & Common name & Family & Life cycle \\
\hline 1 & Parthenium hysterophorus $L$. & Parthenium weed & Asteraceae & A \\
\hline 2 & Taraxicum officinale $L$. & Com. Dandelion & Asteraceae & $\mathrm{P}$ \\
\hline 3 & Silybum marianum L. & Milk thistle & Asteraceae & A \\
\hline 4 & Cyperus rotundus $L$. & Purple nut sedge & Cyperaceae & A \\
\hline 5 & Cynodon dactylon L. & Bermuda grass & Poaceae & $\mathrm{P}$ \\
\hline 6 & Euphorbia helioscopia L. & Mad women's & Euphorbiaceae & A \\
\hline 7 & Poa annua $L$. & Annual blue grass & Poaceae & A \\
\hline 8 & Sorghum helephense L. & Johnson grass & Poaceae & $\mathrm{P}$ \\
\hline 9 & Conyza spp L. & Canadian horse & Asteraceae & A \\
\hline 10 & Plantago lenceulata $L$. & Rebwort & Plantaginaceae & A \\
\hline 11 & Convolvulus arvensis $L$. & Field bind weed & Convolulaceae & $\mathrm{P}$ \\
\hline 12 & Rumex crispus $L$. & Curly dock & Polygonaceae & $P$ \\
\hline 13 & Broussonetia papyrifera & Paper mulbary & Moraceae & $\mathrm{P}$ \\
\hline 14 & Sonchus oleraceus L. & Sow thistle & Asteraceae & A \\
\hline 15 & Xanthium strumarium & Common & Asteraceae & $A$ \\
\hline 16 & Melilotus parviflora & Sweet-clover & Fabaceae & A \\
\hline 17 & Anagallis arvensis $L$. & Chick weed & Primulaceae & $A$ \\
\hline 18 & Oxalis corymbosa $L$. & Wood sorrel & Oxalidaceae & $A$ \\
\hline 19 & Cronopus didimus $L$. & Swinecress & Brassicaceae & A \\
\hline 20 & Dicanthium annulatum & Ring beard grass & Poaceae & $A$ \\
\hline 21 & Calendula arvensis L. & Field mari gold & Asteraceae & A \\
\hline 22 & Malvestrum spp. & Mallow & Malvaceae & A \\
\hline 23 & Ranunculus spp. & Butter cup & Rananculaceae & A \\
\hline 24 & Ammi visnaga $L$. & Besnaga & Apiaceae & A \\
\hline 25 & Amaranthus viridis $L$. & Slender Amaranth & Amaranthaceae & A \\
\hline 26 & Medicago denticulate $L$. & Burclover & Fabaceae & A \\
\hline 27 & Chenepodium album $L$. & Common lambs- & Chenopodiaceae & A \\
\hline 28 & Alternanthera denticulate $R \cdot B r$ & Lesser joy weed & Amaranthaceae & $\mathrm{P}$ \\
\hline 29 & Eleusine indica $L$. & Goose grass & Poaceae & A \\
\hline 30 & Datura alba L. & Jimson weed & Solanaceae & $\mathrm{P}$ \\
\hline 31 & Fumaria indica $L$. & Common & Papaveraceae & A \\
\hline 32 & Dactyloctenium aegyptium L. & Coaster finger & Poaceae & $A$ \\
\hline
\end{tabular}

$A=$ Annual and $P=$ perennial

Table 2 Absolute weeds density for the selected site for Peshawar University Campus.

\begin{tabular}{|c|c|c|c|c|c|c|c|c|c|c|c|}
\hline \multirow[t]{2}{*}{ Weed Species Botanical Names } & \multirow{2}{*}{$\begin{array}{c}\text { Weed Species Common } \\
\text { Names }\end{array}$} & \multicolumn{4}{|c|}{ The University of Agriculture } & \multicolumn{4}{|c|}{ University of Peshawar } & \multicolumn{2}{|c|}{$\begin{array}{l}\text { Islamia College } \\
\text { University }\end{array}$} \\
\hline & & D.F.A & A.F.A & P.B.G.F & H.F.A & N.A.B & F.I.C & F.I.J & D.G. & I.C.S & I.C.U. \\
\hline Parthenium hysterophorus $L$. & Parthenium weed & 76.8 & 59.2 & 44.8 & 50.4 & 58.4 & 41.6 & 42.4 & 32 & 38.4 & 36.8 \\
\hline Taraxicum officinale $L$. & Common dandelion & 10.4 & 1.6 & 0 & 24.8 & 0 & 12.8 & 12 & 2.4 & 0 & 0 \\
\hline Silybum marianum L. & Milk thistle & 18.4 & 0 & 0 & 0 & 0 & 0 & 0 & 0 & 0 & 0 \\
\hline Cyperus rotundus $L$. & Purple nut sedge & 16.8 & 0 & 0 & 15.2 & 32.8 & 0 & 4.8 & 0 & 0 & 0 \\
\hline Cynodon dactylon $L$. & Bermuda grass & 12.8 & 55.2 & 41.6 & 24 & 28 & 80 & 46.4 & 100 & 64 & 53.6 \\
\hline Euphorbia helioscopia L. & Mad women's & 8.8 & 12 & 8.8 & 5.6 & 0 & 0 & 0 & 8.8 & 0 & 0 \\
\hline Poa annua $L$. & Annual blue grass & 7.2 & 6.3 & 0 & 8 & 0 & 0 & 4 & 0 & 0 & 0 \\
\hline Sorghum helephense $L$. & Johnson grass & 7.2 & 10.4 & 8 & 0 & 24 & 0 & 0 & 0 & 0 & 0 \\
\hline Conyza spp $L$. & Canadian horse & 0 & 14.4 & 33.6 & 0 & 0 & 0 & 0 & 3.2 & 0 & 0 \\
\hline Plantago lenceulata $L$. & Rebwort & 0 & 5.6 & 0 & 8 & 2.4 & 6.4 & 0 & 5.6 & 0 & 0 \\
\hline Convolvulus arvensis $L$. & Field bind weed & 0 & 10.4 & 13.6 & 0 & 7.2 & 0 & 20.8 & 5.6 & 7.2 & 4 \\
\hline Rumex crispus $L$. & Curly dock & 0 & 1.6 & 0 & 0 & 0 & 3.2 & 0 & 7.2 & 15.2 & 20.8 \\
\hline Broussonetia papyrifera & Paper mulbary & 0 & 1.6 & 0 & 0 & 0 & 0 & 0 & 0 & 0 & 0 \\
\hline Sonchus oleraceus L. & Sow thistle & 0 & 1.6 & 0 & 0 & 0 & 12.8 & 0 & 0 & 3.2 & 0 \\
\hline Xanthium strumarium & Common & 0 & 6.4 & 0 & 0.8 & 0 & 0 & 0 & 0 & 0 & 0 \\
\hline Melilotus parviflora & Sweet-clover & 0 & 4.8 & 0 & 0 & 0 & 0 & 0 & 0 & 0 & 0 \\
\hline
\end{tabular}




\begin{tabular}{|c|c|c|c|c|c|c|c|c|c|c|c|}
\hline \multirow[t]{2}{*}{ Weed Species Botanical Names } & \multirow{2}{*}{$\begin{array}{c}\text { Weed Species Common } \\
\text { Names }\end{array}$} & \multicolumn{4}{|c|}{ The University of Agriculture } & \multicolumn{4}{|c|}{ University of Peshawar } & \multicolumn{2}{|c|}{$\begin{array}{l}\text { Islamia College } \\
\text { University }\end{array}$} \\
\hline & & D.F.A & A.F.A & P.B.G.F & H.F.A & N.A.B & F.I.C & F.I.J & D.G. & I.C.S & I.C.U. \\
\hline Anagallis arvensis $L$. & Chick weed & 0 & 2.4 & 0 & 0 & 0 & 0 & 0 & 0 & 0 & 0 \\
\hline Oxalis corymbosa $L$. & Wood sorrel & 0 & 0 & 24.8 & 0 & 0 & 32.8 & 0 & 9.6 & 37.6 & 0 \\
\hline Cronopus didimus L. & Swinecress & 0 & 0 & 3.2 & 0 & 0 & 0 & 0 & 0 & 0 & 0 \\
\hline Dicanthium annulatum & Ring beard grass & 0 & 0 & 0 & 6.4 & 12.8 & 0 & 0 & 0 & 0 & 17.6 \\
\hline Calendula arvensis $L$. & Field mari gold & 0 & 0 & 0 & 0 & 0 & 7.2 & 0 & 0 & 9.6 & 0 \\
\hline Malvestrum spp. & Mallow & 0 & 0 & 0 & 0 & 0 & 2.4 & 4 & 11.2 & 0 & 0 \\
\hline Ranunculus spp. & Butter cup & 0 & 0 & 0 & 0 & 0 & 22.4 & 0 & 0 & 18.4 & 4.8 \\
\hline Ammi visnaga $L$. & Besnaga & 0 & 0 & 0 & 0 & 0 & 6.4 & 12 & 0 & 0 & 0 \\
\hline Amaranthus viridis $L$. & Slender Amaranth & 0 & 0 & 0 & 0 & 0 & 0 & 3.2 & 0 & 0 & 0 \\
\hline Medicago denticulate $L$. & Burclover & 0 & 0 & 0 & 0 & 0 & 0 & 0 & 20 & 12.8 & 0 \\
\hline Chenepodium album $L$. & Common lambs- & 0 & 0 & 0 & 0 & 0 & 0 & 0 & 3.2 & 0 & 0 \\
\hline Alternanthera denticulate & Lesser joy weed & 0 & 0 & 0 & 0 & 0 & 0 & 0 & 4 & 0 & 0 \\
\hline Eleusine indica $L$. & Goose grass & 0 & 0 & 0 & 0 & 0 & 0 & 0 & 0 & 31.2 & 9.6 \\
\hline Datura alba $L$. & Jimson weed & 0 & 0 & 0 & 0 & 0 & 0 & 0 & 0 & 5.6 & 0 \\
\hline Fumaria indica $L$. & Common & 0 & 0 & 0 & 0 & 0 & 0 & 0 & 0 & 10.4 & 0 \\
\hline Dactyloctenium aegyptium L. & Coaster finger & 0 & 0 & 0 & 0 & 5.6 & 0 & 0 & 0 & 0 & 0 \\
\hline
\end{tabular}

(33.44). Whereas, the depressed percent of relative density of Parthenium (15.0\%) was infested at the Islamia Collegiate School. The means data further showed the Cynodon dactylon L. was the more occurred species with $36.41 \%$ relative density and the Parthenium weed $25.00 \%$ and Oxylis Species $4.5 \%$. In studied survey, observed the Parthenium distribution become more rapidly day by day due to its strong competitive nature. After Cynodon dactylon, the density of Parthenium is high as compare to others infested flora, it can be in non-cropping area as well as crop cultivated area. The dominancy of Parthenium weeds on local flora, due to the rapid infestation in wastelands or undisturb area. May be due to its invasive potential, high growth rate, more allelopathic properties, large quantity of seeds production and short growth cycle [22]. Same situation was surveyed, in rainy season the distribution and infestation of Parthenium and Achyranthes aspera L. weed are more dominant [23] (Table 3).

\section{Absolute frequency (\%)}

The configuration of local flora in the studied locations at the Peshawar University Campus and the New Developmental Farm, The University of Agriculture Peshawar and Islamia College University. In absolute frequency of local flora, the higher frequency $100 \%$ of Parthenium weed was noted at the studied areas, the University of Peshawar and The University of Agriculture and Islamia College University. In spite of fact that, the lower absolute frequency Parthenium occurred in Agronomy field area $80 \%$ were absorbed and then Cynodon dactylon L., Taraxicum officinale $L$. and Convolvulus arvensis $L$. as the subsequent evenly infested species in the studied location. The absolute Frequency mean data of flora distribution showed the Parthenium and Johnson grass as more frequent weeds and then Euphorbia heliscopia, Poa annua, and Plantago lenceulata respectively. The Parthenium is an alien weed, commonly distributed in neotropical and pan-tropical distributed, Pakistan. The absolute frequency and spreading is very fast in degraded areas, wetlands, along the water bodies, railway tracks and roadsides as well as nowadays reported in crops cultivated field. Also, have negative effects on animal husbandry, crop production and native flora as well human health [24] (Table 4).

\section{Relative frequency (\%)}

The distributed weeds relative frequency is a good statistic that indicates the infestation of weeds in the investigated location. The mean data of relative frequency show that the domination of Parthenium hysterophorus L. (20.28\%) was calculated followed by Cynodon dactylon L. (15.55\%) and Taraxicum officinale L. (5.92\%) across all sites. The highest relative frequency of Parthenium occurred in Islamia College University area which is (25\%) and then Cynodon dactylon L. also occurred in the same site. Tabulated data furthermore illustrates the Parthenium weed distribution was almost same in all the studied sites; though its relative frequency $(12.9 \%)$ was lowest recorded at Agronomy field area (The University of Agriculture Peshawar) where there were a good weed management practices in Agronomy field. Moreover, Chickweed was noted with low (3.22\%) relative frequency at Agronomy field. In survey, it was observe that the Parthenium weed relative frequency is low in cropping area due weed good management practice while, high in non-cropping area due undisturbed area. The highest relative frequency of Parthenium in the non-cropping area are too high as compare to agricultural cropping where always up to some extend weed management practices are in running [19]. This could have been predicted and is most likely due to this weed's highly competitive and allelopathic habit [25]. The displacement of native species may also be due to this weed's highly adaptive nature and because it is poorly grazed by cattle. Similar results to those being reported here for a decline in species richness follow in parthenium weed invasion have also been seen in India and Ethiopia [26,27] (Table 5).

\section{Importance value (\%)}

The distributions of local flora at everywhere, the Importance 
Table 3 Relative weeds density (\%) for the selected sites of Peshawar University Campus.

\begin{tabular}{|c|c|c|c|c|c|c|c|c|c|c|c|}
\hline \multirow{2}{*}{$\begin{array}{c}\text { Weed Species Botanical } \\
\text { Names }\end{array}$} & \multirow{2}{*}{$\begin{array}{l}\text { Weed Species } \\
\text { Common Names }\end{array}$} & \multicolumn{4}{|c|}{ The University of Agriculture } & \multicolumn{4}{|c|}{ University of Peshawar } & \multicolumn{2}{|c|}{$\begin{array}{l}\text { Islamia College } \\
\text { University }\end{array}$} \\
\hline & & D.F.A. & A.F.A. & P.B.G.F & H.F.A & N.A.B & F.I. C & F.I.J & D.G. & I.C.S. & I.C.U \\
\hline Parthenium hysterophorus $L$. & Parthenium weed & 48.48 & 30.58 & 25.11 & 35.2 & 33.3 & 17.39 & 25.2 & 15.04 & 15.14 & 25 \\
\hline Taraxicum officinale $L$. & Com. Dandelion & 6.57 & 0.83 & 0 & 17.3 & 0 & 5.35 & 7.14 & 1.13 & 0 & 0 \\
\hline Silybum marianum L. & Milk thistle & 11.62 & 0 & 0 & 0 & 0 & 0 & 0 & 0 & 0 & 0 \\
\hline Cyperus rotundus $L$. & Purple nut sedge & 10.61 & 0 & 0 & 10.6 & 18.7 & 0 & 2.86 & 0 & 0 & 0 \\
\hline Cynodon dactylon L. & Bermuda grass & 8.08 & 28.51 & 23.32 & 16.8 & 15.9 & 33.44 & 27.6 & 46.99 & 25.24 & 36.61 \\
\hline Euphorbia helioscopia L. & Mad women's & 5.56 & 6.19 & 4.93 & 3.91 & 0 & 0 & 0 & 4.14 & 0 & 0 \\
\hline Poa annua $L$. & Annual blue grass & 4.55 & 3.31 & 0 & 5.59 & 0 & 0 & 2.38 & 0 & 0 & 0 \\
\hline Sorghum helephense L. & Johnson grass & 4.55 & 5.37 & 4.48 & 0 & 13.7 & 0 & 0 & 0 & 0 & 0 \\
\hline Conyza spp $L$. & Canadian horse & 0 & 7.44 & 18.83 & 0 & 0 & 0 & 0 & 1.5 & 0 & 0 \\
\hline Plantago lenceulata $L$. & Rebwort & 0 & 2.89 & 0 & 5.59 & 1.37 & 0 & 0 & 2.63 & 0 & 0 \\
\hline Convolvulus arvensis $L$. & Field bind weed & 0 & 5.37 & 7.62 & 0 & 4.11 & 2.68 & 12.3 & 2.63 & 2.84 & 2.72 \\
\hline Rumex crispus $L$. & Curly dock & 0 & 0.83 & 0 & 0 & 0 & 1.34 & 0 & 3.38 & 5.99 & 14.13 \\
\hline Broussonetia papyrifera & Paper mulbary & 0 & 0.83 & 0 & 0 & 0 & 0 & 0 & 0 & 0 & 0 \\
\hline Sonchus oleraceus L. & Sow thistle & 0 & 0.83 & 0 & 0 & 0 & 5.35 & 0 & 0 & 1.26 & 0 \\
\hline Xanthium strumarium & Common & 0 & 3.31 & 0 & 0.56 & 0 & 0 & 0 & 0 & 0 & 0 \\
\hline Melilotus parviflora & Sweet-clover & 0 & 2.48 & 0 & 0 & 0 & 0 & 0 & 0 & 0 & 0 \\
\hline Anagallis arvensis $L$. & Chick weed & 0 & 1.24 & 0 & 0 & 0 & 0 & 0 & 0 & 0 & 0 \\
\hline Oxalis corymbosa $L$. & Wood sorrel & 0 & 0 & 13.9 & 0 & 0 & 13.71 & 0 & 4.51 & 14.83 & 0 \\
\hline Cronopus didimus $L$. & Swinecress & 0 & 0 & 1.79 & 0 & 0 & 0 & 0 & 0 & 0 & 0 \\
\hline Dicanthium annulatum & Ring beard grass & 0 & 0 & 0 & 4.47 & 7.31 & 4.68 & 0 & 0 & 0 & 11.96 \\
\hline Calendula arvensis $L$. & Field mari gold & 0 & 0 & 0 & 0 & 0 & 3.01 & 0 & 0 & 3.79 & 0 \\
\hline Malvestrum spp. & Mallow & 0 & 0 & 0 & 0 & 0 & 1 & 2.38 & 5.26 & 0 & 0 \\
\hline Ranunculus spp. & Butter cup & 0 & 0 & 0 & 0 & 0 & 9.36 & 0 & 0 & 7.26 & 3.2 \\
\hline Ammi visnaga $L$. & Besnaga & 0 & 0 & 0 & 0 & 0 & 2.68 & 7.14 & 0 & 0 & 0 \\
\hline Amaranthus viridis $L$. & Slender Amaranth & 0 & 0 & 0 & 0 & 0 & 0 & 1.9 & 0 & 0 & 0 \\
\hline Medicago denticulate $L$. & Burclover & 0 & 0 & 0 & 0 & 0 & 0 & 0 & 9.4 & 5.05 & 0 \\
\hline Chenepodium album L. & Common lambs- & 0 & 0 & 0 & 0 & 0 & 0 & 0 & 1.5 & 0 & 0 \\
\hline Alternanthera denticulate & Lesser joy weed & 0.00 & 0 & 0 & 0 & 0 & 0 & 0 & 1.88 & 0 & 0 \\
\hline Eleusine indica $L$. & Goose grass & 0 & 0 & 0 & 0 & 0 & 0 & 0 & 0 & 12.3 & 6.52 \\
\hline Datura alba L. & Jimson weed & 0 & 0 & 0 & 0 & 0 & 0 & 0 & 0 & 2.21 & 0 \\
\hline Fumaria indica $L$. & Common & 0 & 0 & 0 & 0 & 0 & 0 & 0 & 0 & 4.1 & 0 \\
\hline Dactyloctenium aegyptium L. & Coaster finger & 0 & 0 & 0 & 0 & 3.2 & 0 & 0 & 0 & 0 & 0 \\
\hline
\end{tabular}

Table 4 Weeds Absolute Frequency (\%) table for the selected sites of Peshawar University campus.

\begin{tabular}{|c|c|c|c|c|c|c|c|c|c|c|c|}
\hline \multirow{2}{*}{$\begin{array}{l}\text { Weed Species Botanical } \\
\text { Names }\end{array}$} & \multirow{2}{*}{$\begin{array}{c}\text { Weed Species Common } \\
\text { Names }\end{array}$} & \multicolumn{4}{|c|}{ The University of Agriculture } & \multicolumn{4}{|c|}{ University of Peshawar } & \multicolumn{2}{|c|}{$\begin{array}{l}\text { Islamia College } \\
\text { University }\end{array}$} \\
\hline & & D.F.A. & A.F.A. & P.B.G.F. & H.F.A. & N.A.B. & F.I. C. & F.I.J. & D.G. & I.C.S. & I.C.U. \\
\hline arthenium hysterophorus $L$. & Parthenium weed & 100 & 80 & 100 & 100 & 100 & 100 & 100 & 100 & 100 & 100 \\
\hline Taraxicum officinale L. & Common dandelion & 60 & 20 & 0 & 80 & 0 & 60 & 40 & 20 & 0 & 0 \\
\hline Silybum marianum L. & Milk thistle & 80 & 0 & 0 & 0 & 0 & 0 & 0 & 0 & 0 & 0 \\
\hline Cyperus rotundus $L$. & Purple nut sedge & 60 & 0 & 0 & 60 & 100 & 0 & 20 & 0 & 0 & 0 \\
\hline Cynodon dactylon $L$. & Bermuda grass & 40 & 100 & 80 & 60 & 60 & 80 & 60 & 100 & 80 & 100 \\
\hline Euphorbia helioscopia L. & Mad womems & 40 & 20 & 20 & 40 & 0 & 0 & 0 & 40 & 0 & 0 \\
\hline Poa annua $L$. & Annualblue grass & 40 & 20 & 0 & 20 & 0 & 0 & 20 & 0 & 0 & 0 \\
\hline Sorghum helephense $L$. & Johnson grass & 40 & 60 & 40 & 0 & 100 & 0 & 0 & 0 & 0 & 0 \\
\hline Conyza spp $L$. & Canadian horse & 0 & 60 & 80 & 0 & 0 & 0 & 0 & 20 & 0 & 0 \\
\hline Plantago lenceulata $L$. & Rebwort & 0 & 60 & 0 & 40 & 20 & 20 & 0 & 20 & 0 & 0 \\
\hline Convolvulus arvensis $L$. & Field bind weed & 0 & 60 & 80 & 0 & 40 & 0 & 80 & 40 & 40 & 20 \\
\hline Rumex crispus $L$. & Curly dock & 0 & 20 & 0 & 0 & 0 & 20 & 0 & 40 & 60 & 80 \\
\hline Broussonetia papyrifera & Paper mulbary & 0 & 20 & 0 & 0 & 0 & 0 & 0 & 0 & 0 & 0 \\
\hline Sonchus oleraceus L. & Sow thistle & 0 & 20 & 0 & 0 & 0 & 40 & 0 & 0 & 20 & 0 \\
\hline Xanthium strumarium & Common & 0 & 20 & 0 & 20 & 0 & 0 & 0 & 0 & 0 & 0 \\
\hline
\end{tabular}




\begin{tabular}{|c|c|c|c|c|c|c|c|c|c|c|c|}
\hline \multirow{2}{*}{$\begin{array}{l}\text { Weed Species Botanical } \\
\text { Names }\end{array}$} & \multirow{2}{*}{$\begin{array}{c}\text { Weed Species Common } \\
\text { Names }\end{array}$} & \multicolumn{4}{|c|}{ The University of Agriculture } & \multicolumn{4}{|c|}{ University of Peshawar } & \multicolumn{2}{|c|}{$\begin{array}{c}\text { Islamia College } \\
\text { University }\end{array}$} \\
\hline & & D.F. A. & A.F.A. & P.B.G.F. & H.F.A. & N.A.B. & F.I. C. & F.I.J. & D.G. & I.C.S. & I.C.U. \\
\hline Melilotus parviflora & Sweet-clover & 0 & 40 & 0 & 0 & 0 & 0 & 0 & 0 & 0 & 0 \\
\hline Anagallis arvensis $L$. & Chick weed & 0 & 20 & 0 & 0 & 0 & 0 & 0 & 0 & 0 & 0 \\
\hline Oxalis corymbosa $L$. & Wood sorrel & 0 & 0 & 60 & 0 & 0 & 40 & 0 & 20 & 40 & 0 \\
\hline Cronopus didimus L. & Swinecress & 0 & 0 & 20 & 0 & 0 & 0 & 0 & 0 & 0 & 0 \\
\hline Dicanthium annulatum & Ring beard grass & 0 & 0 & 0 & 20 & 40 & 40 & 0 & 0 & 0 & 60 \\
\hline Calendula arvensis $L$. & Field mari gold & 0 & 0 & 0 & 0 & 0 & 20 & 0 & 0 & 20 & 0 \\
\hline Malvestrum spp. & Mallow & 0 & 0 & 0 & 0 & 0 & 20 & 40 & 40 & 0 & 0 \\
\hline Ranunculus spp. & Butter cup & 0 & 0 & 0 & 0 & 0 & 40 & 0 & 0 & 40 & 20 \\
\hline Ammi visnaga $L$. & Besnaga & 0 & 0 & 0 & 0 & 0 & 20 & 40 & 0 & 0 & 0 \\
\hline Amaranthus viridis $L$. & Slender Amaranth & 0 & 0 & 0 & 0 & 0 & 0 & 20 & 0 & 0 & 0 \\
\hline Medicago denticulate $L$. & Burclover & 0 & 0 & 0 & 0 & 0 & 0 & 0 & 20 & 20 & 0 \\
\hline Chenepodium album L. & Common lambs- & 0 & 0 & 0 & 0 & 0 & 0 & 0 & 20 & 0 & 0 \\
\hline Alternanthera denticulate & Lesser joy weed & 0 & 0 & 0 & 0 & 0 & 0 & 0 & 20 & 0 & 0 \\
\hline Eleusine indica $L$. & Goose grass & 0 & 0 & 0 & 0 & 0 & 0 & 0 & 0 & 80 & 20 \\
\hline Datura alba L. & Jimson weed & 0 & 0 & 0 & 0 & 0 & 0 & 0 & 0 & 40 & 0 \\
\hline Fumaria indica $L$. & Common & 0 & 0 & 0 & 0 & 0 & 0 & 0 & 0 & 20 & 0 \\
\hline Dactyloctenium aegyptium L. & Coaster finger & 0 & 0 & 0 & 0 & 20 & 0 & 0 & 0 & 0 & 0 \\
\hline
\end{tabular}

Table 5 Weeds Relative Frequency (\%) for the selected sites of Peshawar University campus.

\begin{tabular}{|c|c|c|c|c|c|c|c|c|c|c|c|}
\hline \multirow{2}{*}{$\begin{array}{c}\text { Weed Species Botanical } \\
\text { Names }\end{array}$} & \multirow{2}{*}{$\begin{array}{l}\text { Weed Species } \\
\text { Common Names }\end{array}$} & \multicolumn{4}{|c|}{ The University of Agriculture } & \multicolumn{4}{|c|}{ University of Peshawar } & \multicolumn{2}{|c|}{$\begin{array}{l}\text { Islamia College } \\
\text { University }\end{array}$} \\
\hline & & D.F.A. & A.F.A. & P.B.G.F. & H.F.A & N.A.B & F.I. C & F.I.J. & D.G. & I.C.S & I.C.U. \\
\hline Parthenium hysterophorus $L$. & Parthenium weed & 21.74 & 12.9 & 20.83 & 22.73 & 20 & 20 & 21.74 & 20 & 17.86 & 25 \\
\hline Taraxicum officinale L. & Comm. dandelion & 13.04 & 3.23 & 0 & 18.18 & 0 & 12 & 8.7 & 4 & 0 & 0 \\
\hline Silybum marianum L. & Milk thistle & 17.39 & 0 & 0 & 0 & 0 & 0 & 0 & 0 & 0 & 0 \\
\hline Cyperus rotundus $L$. & Purple nut sedge & 13.04 & 0 & 0 & 13.64 & 20 & 0 & 4.35 & 0 & 0 & 0 \\
\hline Cynodon dactylon L. & Bermuda grass & 8.7 & 16.13 & 16.67 & 13.64 & 12 & 16 & 13.04 & 20 & 14.29 & 25 \\
\hline Euphorbia helioscopia L. & Mad women's & 8.7 & 3.23 & 4.17 & 9.09 & 0 & 0 & 0 & 8 & 0 & 0 \\
\hline Poa annua $L$. & Annual blue grass & 8.7 & 3.23 & 0 & 4.55 & 0 & 0 & 4.35 & 0 & 0 & 0 \\
\hline Sorghum helephense L. & Johnson grass & 8.7 & 9.68 & 8.33 & 0 & 20 & 0 & 0 & 0 & 0 & 0 \\
\hline Conyza spp $L$. & Canadian horse & 0 & 9.68 & 16.67 & 0 & 0 & 0 & 0 & 4 & 0 & 0 \\
\hline Plantago lenceulata $L$. & Rebwort & 0 & 9.68 & 0 & 9.09 & 4 & 0 & 0 & 4 & 0 & 0 \\
\hline Convolvulus arvensis $L$. & Field bind weed & 0 & 9.68 & 16.67 & 0 & 8 & 4 & 17.39 & 8 & 7.14 & 5 \\
\hline Rumex crispus $L$. & Curly dock & 0 & 3.23 & 0 & 0 & 0 & 4 & 0 & 8 & 10.71 & 20 \\
\hline Broussonetia papyrifera & Paper mulbary & 0 & 3.23 & 0 & 0 & 0 & 0 & 0 & 0 & 0 & 0 \\
\hline Sonchus oleraceus L. & Sow thistle & 0 & 3.23 & 0 & 0 & 0 & 8 & 0 & 0 & 3.57 & 0 \\
\hline Xanthium strumarium & Common & 0 & 3.23 & 0 & 4.55 & 0 & 0 & 0 & 0 & 0 & 0 \\
\hline Melilotus parviflora & Sweet-clover & 0 & 6.45 & 0 & 0 & 0 & 0 & 0 & 0 & 0 & 0 \\
\hline Anagallis arvensis $L$. & Chick weed & 0 & 3.23 & 0 & 0 & 0 & 0 & 0 & 0 & 0 & 0 \\
\hline Oxalis corymbosa $L$. & Wood sorrel & 0 & 0 & 12.5 & 0 & 0 & 8 & 0 & 4 & 7.14 & 0 \\
\hline Cronopus didimus L. & Swinecress & 0 & 0 & 4.17 & 0 & 0 & 0 & 0 & 0 & 0 & 0 \\
\hline Dicanthium annulatum & Ring beard grass & 0 & 0 & 0 & 4.55 & 8 & 8 & 0 & 0 & 0 & 15 \\
\hline Calendula arvensis $L$. & Field mari gold & 0 & 0 & 0 & 0 & 0 & 4 & 0 & 0 & 3.57 & 0 \\
\hline Malvestrum spp. & Mallow & 0 & 0 & 0 & 0 & 0 & 4 & 8.7 & 8 & 0 & 0 \\
\hline Ranunculus spp. & Butter cup & 0 & 0 & 0 & 0 & 0 & 8 & 0 & 0 & 7.14 & 5 \\
\hline Ammi visnaga $L$. & Besnaga & 0 & 0 & 0 & 0 & 0 & 4 & 8.7 & 0 & 0 & 0 \\
\hline Amaranthus viridis $L$. & Slender Amaranth & 0 & 0 & 0 & 0 & 0 & 0 & 4.35 & 0 & 0 & 0 \\
\hline Medicago denticulate L. & Bur clover & 0 & 0 & 0 & 0 & 0 & 0 & 0 & 4 & 3.57 & 0 \\
\hline Chenepodium album L. & Common lambs- & 0 & 0 & 0 & 0 & 0 & 0 & 0 & 4 & 0 & 0 \\
\hline Alternanthera denticulate & Lesser joy weed & 0 & 0 & 0 & 0 & 0 & 0 & 0 & 4 & 0 & 0 \\
\hline Eleusine indica $L$. & Goose grass & 0 & 0 & 0 & 0 & 0 & 0 & 0 & 0 & 14.29 & 5 \\
\hline Datura alba L. & Jimson weed & 0 & 0 & 0 & 0 & 0 & 0 & 0 & 0 & 7.14 & 0 \\
\hline Fumaria indica $L$. & Common & 0 & 0 & 0 & 0 & 0 & 0 & 0 & 0 & 3.57 & 0 \\
\hline Dactyloctenium aegyptium L. & Coaster finger & 0 & 0 & 0 & 0 & 4 & 0 & 0 & 0 & 0 & 0 \\
\hline
\end{tabular}


Table 6 Weeds Importance Value (IV) for the selected sites of Peshawar University campus.

\begin{tabular}{|c|c|c|c|c|c|c|c|c|c|c|c|}
\hline \multirow{2}{*}{$\begin{array}{c}\text { Weed Species Botanical } \\
\text { Names }\end{array}$} & \multirow{2}{*}{$\begin{array}{l}\text { Weed Species } \\
\text { Common Names }\end{array}$} & \multicolumn{4}{|c|}{ The University of Agriculture } & \multicolumn{4}{|c|}{ University of Peshawar } & \multicolumn{2}{|c|}{$\begin{array}{l}\text { Islamia College } \\
\text { University }\end{array}$} \\
\hline & & D.F.A. & A.F.A. & P.B.G.F & H.F.A. & N.A.B. & F.I. C. & F.I.J. & D.G. & I.C.S. & I.C.U. \\
\hline Parthenium hysterophorus $L$. & Parthenium weed & 45.98 & 28.19 & 33.39 & 0.33 & 36.67 & 28.7 & 34.36 & 27.52 & 25.43 & 37.5 \\
\hline Taraxicum officinale $L$. & Common dandelion & 16.33 & 3.64 & 0 & 6.84 & 0 & 14.68 & 12.27 & 4.56 & 0 & 0 \\
\hline Silybum marianum $L$. & Milk thistle & 23.2 & 0 & 0 & 0 & 0 & 0 & 0 & 0 & 0 & 0 \\
\hline Cyperus rotundus $L$. & Purple nut sedge & 18.35 & 0 & 0 & 8.94 & 29.36 & 0 & 5.78 & 0 & 0 & 0 \\
\hline Cynodon dactylon L. & Bermuda grass & 12.74 & 30.38 & 28.33 & 2.02 & 19.19 & 32.72 & 26.85 & 43.5 & 26.9 & 43.21 \\
\hline Euphorbia helioscopia L. & Mad women's & 11.47 & 6.32 & 6.63 & 1.05 & 0 & 0 & 0 & 10.07 & 0 & 0 \\
\hline Poa annua $L$. & Annual blue grass & 10.97 & 4.88 & 0 & 7.34 & 0 & 0 & 5.54 & 0 & 0 & 0 \\
\hline Sorghum helephense L. & Johnson grass & 10.97 & 12.36 & 10.58 & 0 & 26.85 & 0 & 0 & 0 & 0 & 0 \\
\hline Conyza spp L. & Canadian horse & 0 & 13.4 & 26.08 & 0 & 0 & 0 & 0 & 4.75 & 0 & 0 \\
\hline Plantago lenceulata $L$. & Reb worth & 0 & 11.12 & 0 & 1.88 & 4.68 & 0 & 0 & 5.32 & 0 & 0 \\
\hline Convolvulus arvensis $L$. & Field bind weed & 0 & 12.36 & 20.48 & 0 & 10.05 & 5.34 & 23.58 & 9.32 & 8.56 & 6.36 \\
\hline Rumex crispus $L$. & Curly dock & 0 & 3.64 & 0 & 0 & 0 & 4.67 & 0 & 9.69 & 13.71 & 27.07 \\
\hline Broussonetia papyrifera & Paper mulberry & 0 & 3.64 & 0 & 0 & 0 & 0 & 0 & 0 & 0 & 0 \\
\hline Sonchus oleraceus $L$. & Sow thistle & 0 & 3.64 & 0 & 0 & 0 & 10.68 & 0 & 0 & 4.2 & 0 \\
\hline Xanthium strumarium & Common & 0 & 4.88 & 0 & 4.82 & 0 & 0 & 0 & 0 & 0 & 0 \\
\hline Melilotus parviflora & Sweet-clover & 0 & 7.69 & 0 & 0 & 0 & 0 & 0 & 0 & 0 & 0 \\
\hline Anagallis arvensis $L$. & Chick weed & 0 & 3.85 & 0 & 0 & 0 & 0 & 0 & 0 & 0 & 0 \\
\hline Oxalis corymbosa $L$. & Wood sorrel & 0 & 0 & 19.45 & 0 & 0 & 14.86 & 0 & 6.26 & 14.56 & 0 \\
\hline Cronopus didimus $L$. & Swine cress & 0 & 0 & 5.06 & 0 & 0 & 0 & 0 & 0 & 0 & 0 \\
\hline Dicanthium annulatum & Ring beard grass & 0 & 0 & 0 & 6.78 & 11.65 & 10.34 & 0 & 0 & 0 & 20.98 \\
\hline Calendula arvensis $L$. & Field marigold & 0 & 0 & 0 & 0 & 0 & 5.51 & 0 & 0 & 5.46 & 0 \\
\hline Malvestrum spp. & Mallow & 0 & 0 & 0 & 0 & 0 & 4.5 & 9.89 & 10.63 & 0 & 0 \\
\hline Ranunculus spp. & Butter cup & 0 & 0 & 0 & 0 & 0 & 12.68 & 0 & 0 & 10.77 & 6.63 \\
\hline Ammi visnaga $L$. & Besnaga & 0 & 0 & 0 & 0 & 0 & 5.34 & 12.27 & 0 & 0 & 0 \\
\hline Amaranthus viridis $L$. & Slender Amaranth & 0 & 0 & 0 & 0 & 0 & 0 & 5.3 & 0 & 0 & 0 \\
\hline Medicago denticulate $L$. & Bur clover & 0 & 0 & 0 & 0 & 0 & 0 & 0 & 8.7 & 6.1 & 0 \\
\hline Chenepodium album L. & Common lambs- & 0 & 0 & 0 & 0 & 0 & 0 & 0 & 4.75 & 0 & 0 \\
\hline Alternanthera denticulate & Lesser joy weed & 0 & 0 & 0 & 0 & 0 & 0 & 0 & 4.94 & 0 & 0 \\
\hline Eleusine indica $L$. & Goose grass & 0 & 0 & 0 & 0 & 0 & 0 & 0 & 0 & 20.4 & 8.26 \\
\hline Datura alba $L$. & Jimson weed & 0 & 0 & 0 & 0 & 0 & 0 & 0 & 0 & 8.25 & 0 \\
\hline Fumaria indica $L$. & Common & 0 & 0 & 0 & 0 & 0 & 0 & 0 & 0 & 5.62 & 0 \\
\hline Dactyloctenium aegyptium L. & Coaster finger & 0 & 0 & 0 & 0 & 5.6 & 0 & 0 & 0 & 0 & 0 \\
\hline
\end{tabular}

Values of that play a key role. Among the studied locations, the mean of all the studied site the minimum (33.81\%) IV was recorded for Parthenium followed by Cynodon dactylon (28.58\%). Apart from that, the maximum Importance Value of 45.98\% for Parthenium was founded at a Dairy farm and then $40.33 \%$ at horticulture field's area. Moreover, in the case of other than Parthenium weed, the Cynodon dactylon and Convolvulus arvensis were ranked as uppermost species in Islamia College and Forest Institute jungle as well as other all 10 studied areas. The collected data defecates the spreading of Parthenium weed in the studied locations, and its successful adjustable nature and it is quite inevitable to monitor the further infestation from infested to uninfected locations. Recently, majority of the rangelands and crops cultivated areas are infested with this invasive, noxious Parthenium weed, due to its fast growth and easily adjustability and dominancy on native flora [28]. The dormancy figure and fast infestation of Parthenium weed in forage, deceased up to $90 \%$. Moreover, the presence of especially invasive weeds make land infertile and reduced the quality of grazing land, animal health, milk products as well meat $[29,30]$. $P$. hysterphorus had become a curse for the natural herbs of the Chhattisgarh plains in India. In Islamabad, the Cannabis sativa is exotic invasive species, was once the most dominant weed species among the herbaceous flora [31]. It is now being replaced by $P$. hysterophorus, which is a 
very versatile species that exhibits characteristics such as strong Allelopathic effect and fast growing ratio [32] (Table 6).

\section{Conclusions}

The Parthenium hysterophorus $L$. weed become more problematic and replaces maximum native flora in the non-crop and

\section{References}

1 Navie SC, McFadyen RE, Panetta FD, Adkins SW (1996) The biology of Australian weeds. Plant Prot Quart 11: 76-88.

2 Auld BA, Hosking J, McFadyen RE (1983) Analysis of the spread of tiger pear and Parthenium weed in Australia. Aus J of Weed Sci 2 : 56-60.

3 Butler JE (1984) Longevity of Parthenium hysterophorus L. seed in the soil. Aus J of Weed Sci 3:6.

4 Navie SC, Priest TE, McFadyen RE, Adkins SW (1998) Efficacy of the stem-gallingmothEpiblemastrenuanaWalk:(Lepidoptera:Tortricidae) as a biologicalcontrol agent for Ragweed Parthenium (Parthenium hysterophorus L). Biol Control 13: 1-8.

5 Dale IJ (1981) Parthenium weed in the Americas. Aust Weeds 1: 8-14.

6 Williams JD, Groves RH (1980) The influence of temperature and photoperiod on growth and developments of Parthenium hysterophorus L. J Weed Res 20: 47-52.

7 Dogra KS, Sood SK, Sharma R (2011) Distribution, Biology, and Ecology of Parthenium hysterophorus L. (Congress Grass) an invasive species in the North-Western Indian Himalaya (Himachal Pradesh). Afri J Plant Science 5: 682-687.

8 Chandras GS, Vartak VD (1970) Symposium on problems caused by Parthenium hysterophorus in Maharashtra Region, India. PANS 16 212-214.

9 Nath R (1981) Note on the effect of Parthenium extract on seed germination and seedling growth in crops. Ind J Agric Sci 51: 601-603.

10 Njoroge JM (1989) Glyphosate (Round-up 36\% a.i.) low rate on annual weeds in Kenya coffee. Ken Coff 54: 713-716.

11 Tamado T, Ohlander L, Milberg P (2002) Interference by the weed Parthenium hysterophorus $\mathrm{L}$. with grain sorghum: influence of weed density and duration of competition. Intl. J Pest Manag 48: 183-188.

12 Picman AK, Towers GHN (1982) Sesquiterpene lactones in various populations of Parthenium hysterophorus. Biochem Systematics and Eco 10: 145-153.

13 Shabbir A (2002) Parthenium hysterophorus L. An exotic weed threatening agricultural lands and biodiversity of Islamabad and adjoining districts. Master's thesis. University of the Punjab, Lahore.

14 Oudhia P (2000) Medicinal herbs and insects of Chhattisgarh India. J Insect Environ 6: 138

15 Khosla SN, Sobti SN (1979) Parthenium - A national health hazard, its controland utility - a review. Pesticides 13: 121-127.

16 Chippendale JF, Panetta FD (1994) The cost of Parthenium weed to the Queensland cattle industry. Plant Prot Quart 9: 73-76.

17 Holman DJ (1981) Parthenium weed threatens Bowen Shire. Queensland Agric J 107: 57-60. cultivated areas. Due to no proper management and high growth rate, nowadays it becomes as key invasive weed of the KPK and Punjab province. So, the proper management of Parthenium weed requires the call attention from the Government, Policy Makers and Agricultural researcher to conserve the local flora and to secure field crops from its infestation to achieve maximum crop yield.

18 Navie SC, Panetta FD, McFadyen RE, Adkins SW (2004) Germinable soil seed banks of central Queensland rangelands invaded by the exotic weed P. hysterophorus L. Weed Biol Manag 4: 154-167.

19 Hussain F, Murad A, Durrani MJ (2004) Weed communities in wheat fields of Mastuj, district Chitral, Pakistan. Pak J Weed Sci Res 10: 101-108.

20 Batish DR, Singh HP, Pandher JK, Arora V, Kohli RK (2002) Phytotoxic effect of Parthenium residues on the selected soil properties and growth of chickpea and radish. Weed Biol Manag 2: 73-78.

21 Singh HP, Batish DR, Pandher JK, Kohli RK (2005) Phytotoxic effects of Parthenium hysterophorus residues on three Brssica species. Weed Biol Manag 5: 105-109.

22 Evans HC (1997) Parthenium hysterophorus: a review of its weed status and the possibilities for biological control. Biocont News Inform 18: 89-98.

23 Oudhia P (2001) Phytosociological Survey of Rainy Season Weeds of Wastelands with Special Reference to Patheniumhysterophorus $L$. in Raipur District, India.

24 Shabbir A, Bajwa R (2007) Parthenium invasion in Pakistan- A. Threat still unrecognized. Pak J Bot 39: 2519-2526.

25 Nguyen T, Bajwa AA, Belgeri A, Navie S, O'Donnell C, et al. (2017) Impact of an invasive weed, Parthenium hysterophorus, on a pasture community in south east Queensland, Australia. Environ Sci Pollut Res 35: 27188-27200.

26 Kohli RK, Dogra KS, Batish DR, Singh HP (2004) Impact of invasive plants on the structure and composition of natural vegetation of Northwestern Indian Himalayas. Weed Technol 18: 1296-1300

27 Nigatu L, Hassen A, Sharma J, Adkins SW (2010) Impact of Parthenium hysterophorus on grazing land communities in north-eastern Ethiopia. Weed Biol Manage 10: 143-152.

28 Tamado T, Milberg P (2000) Weed flora in arable fields of Eastern Ethiopia with emphasis on the occurrence of Parthenium hysterophorus L. J Weed Res 40: 507-521.

29 Bhawmilk PC, Sarkar D (2005) Parthenium hysterophorus: Its world status and potential management. Proceeding of the Second International Conference on Parthenium Management 6: 1-6.

30 Rezene F, Chichaybelu M, Hailegiorgis M (2005) Spread and ecological consequences of Arem 6: 11-23.

31 Mahadevapa M, Das TK, Kumar A (2001) Parthenium: A curse for natural herbs. In: National Research Seminar on Herbal Conservation, Cultivation, Marketing and Utilization with Special Emphasis on Chhattisgarh.

32 Shabbir A, Bajwa R (2006) Distribution of parthenium weed (Parthenium hysterophorus L.), an alien invasive weed species threatening the biodiversity of Islamabad. J Weed Bio Manag 6: 89-95. 